Biomedical Optics

\title{
Quantitative Phase Imaging in Biomedicine
}

Gabriel Popescu YongKeun Park

\section{SPIE.}




\title{
Quantitative Phase Imaging in Biomedicine
}

\author{
Gabriel Popescu \\ University of Illinois at Urbana-Champaign \\ Beckman Institute for Advanced Science and Technology \\ Department of Electrical and Computer Engineering \\ Quantitative Light Imaging Laboratory \\ Urbana, Illinois 61801, United States \\ E-mail: gpopescu@illinois.edu \\ YongKeun Park \\ Korea Advanced Institute of Science and Technology \\ Department of Physics \\ Daejeon 305-701, Republic of Korea \\ E-mail: yk.park@kaist.ac.kr
}

Quantitative phase imaging (QPI) enables label-free quantitative assessment of biological specimens. Over the past decade, we have witnessed tremendous growth in both method development and applications of QPI to biomedicine. Because it is not limited by photobleaching and photoxicity, QPI is capable of studying live specimens over arbitrary time scales and, thus, pursue important biological questions that are otherwise difficult to tackle with current optical imaging techniques. This rapidly emerging field enables the investigation of cells and tissues in terms of morphology and dynamics with nanoscale sensitivity over temporal scales from milliseconds to days and spatial scales from nanometers to centimeters. Quantitative measurements of intrinsic propertiesoptical, chemical, and mechanical-yield new understanding of pathophysiology of cells and tissues.

By combining the principles of microscopy, interferometry, and holography, QPI provides unique capabilities not only for imaging, but also for studying the propagation of optical fields, as well as their interaction with matter. As a result, QPI can be used for noniterative adaptive optics and for measuring light scattering from transparent objects such as single cells. Fourier transform light scattering (FTLS) has been developed as the spatial analog to Fourier transform spectroscopy, essentially using QPI to achieve extreme sensitivity in static and dynamic light scattering measurements. Thus, FTLS has recently bridged imaging and scattering, which traditionally were disciplines placed in different contexts.

The inaugural conference on Quantitative Phase Imaging was held in San Francisco, California, from 7-12 February 2015. The response to the call for papers was absolutely tremendous: 104 papers were presented, including 4 full days of oral presentations, which amounts to one of the largest conferences at Photonics West. Remarkably, the QPI II conference to take place in February 2016 received even more submissions, with the current schedule sitting at 110 papers. These numbers are solid proof that QPI is a field of great current excitement in the research laboratories. Interestingly, this academic interest has been recently paired by fantastic activity in industry: currently there are approximately 10 startup companies operating in the QPI space. These commercial developments are crucial in transferring this novel technology into the hands of biomedical users. In order to stimulate such

(C) 2015 Society of Photo-Optical Instrumentation Engineers (SPIE) activities further, we organized a "Lab to Market" session during the QPI II conference next February, which will bring together visionaries from both well-established and start-up companies.

This special section contains selected papers presented at the conference on Quantitative Phase Imaging, Photonics West 2015, as well as additional contributions submitted in repose to our open call for exciting QPI papers. All submissions were subject to rigorous peer review.

The papers assembled in this special section of the Journal of Biomedical Optics will introduce you to the latest developments and applications in QPI, one the fastest growing fields in biomedical optics. These papers are original research papers, covering topics from imaging and spectroscopy, algorithms and imaging processing, as well as biological and clinical applications. This set of papers presents a cross section of directions in both QPI methods and applications. We hope that this special section will provide a quick update to the established QPI investigator and allow the uninitiated scientist to perhaps gain an interest into this exciting discipline.

The guest editors would also like to express their gratitude to all the contributing authors, as well as to the QPI investigators who are contributing toward the establishment of this emerging field. Special thanks are due to Lihong Wang, Editor-in-Chief of JBO, as well as the JBO staff, in particular Gwen Weerts and Rita Davis.

Gabriel Popescu is associate professor of electrical engineering and bioengineering at University of Illinois at Urbana-Champaign. $\mathrm{He}$ earned a PhD in optics from CREOL and began work on QPI as a postdoctoral associate at MIT's Spectroscopy Laboratory. He is an OSA Fellow and SPIE Fellow. He is an associate editor for Optics Express, Biomedical Optics Express, Scientific Reports, and an editorial board member of the Journal of Biomedical Optics. He founded Phi Optics, Inc., a startup company that commercializes QPI technology for materials life sciences. He wrote the book Quantitative Phase Imaging of Cells and Tissues (McGraw Hill, 2011). To learn more about his research, visit http://light.ece.illinois.edu/

YongKeun (Paul) Park is an associate professor of physics at Korea Advanced Institute of Science and Technology (KAIST), Republic of Korea. He earned a PhD in medical science and medical engineering from Harvard-MIT Health Science and Technology. He has been working on QPI techniques and their applications for the study of pathophysiology of cells and tissues. He is a senior member of SPIE and an editorial board member of Optics Express, Experimental Biology \& Medicine, Scientific Reports and the Journal of Optical Society of Korea. To learn more about his research projects, visit his website: http://bmol.kaist.ac.kr 